\title{
$\gamma \delta$ T cells as early sensors of tissue damage and mediators of secondary neurodegeneration
}

\author{
Mathias Gelderblom *, Priyadharshini Arunachalam and Tim Magnus *
}

Department of Neurology, University Medical Center Hamburg-Eppendorf, Hamburg, Germany

\section{Edited by:}

Arthur Liesz, University Hospital

Munich, Germany

Reviewed by:

Thiruma Arumugam, National

University of Singapore, Singapore

Christopher G. Sobey, Monash

University, Australia

Takashi Shichita, Keio Univeristy,

Japan

*Correspondence:

Mathias Gelderblom and

Tim Magnus, Department of

Neurology, University Medical Center Hamburg-Eppendorf,

Martinistraße 52, 20246 Hamburg,

Germany

e-mail:m.gelderblom@uke.de;

t.magnus@uke.de
Spontaneous or medically induced reperfusion occurs in up to $70 \%$ of patients within $24 \mathrm{~h}$ after cerebral ischemia. Reperfusion of ischemic brain tissue can augment the inflammatory response that causes additional injury. Recently, $T$ cells have been shown to be an essential part of the post-ischemic tissue damage, and especially IL-17 secreting $T$ cells have been implicated in the pathogenesis of a variety of inflammatory reactions in the brain. After stroke, it seems that the innate $\gamma \delta$ T cells are the main IL-17 producing cells and that the $\gamma \delta T$ cell activation constitutes an early and mainly damaging immune response in stroke. Effector mechanism of $\gamma \delta$ T cell derived IL-17 in the ischemic brain include the induction of metalloproteinases, proinflammatory cytokines and neutrophil attracting chemokines, leading to a further amplification of the detrimental inflammatory response. In this review, we will give an overview on the concepts of $\gamma \delta$ T cells and IL-17 in stroke pathophysiology and on their potential importance for human disease conditions.

Keywords: $\gamma \delta$ T cell, stroke, inflammation, IL-17, lymphocyte, brain, ischemia, neutrophils

\section{INTRODUCTION}

Ischemic stroke is the primary reason for sustained disability and the third leading cause of death in the western world. In $85 \%$ of these patients, occlusion of an artery in the brain is the cause of stroke. Early restoration of blood flow (reperfusion) remains the treatment of choice for limiting brain injury following stroke. The reperfusion, which enhances the oxygen and glucose content in the tissue also increases an inflammatory response (Iadecola and Anrather, 2011). The idea that inflammation causes further brain injury is supported by a large number of reports that describe a reduction in infarct size and brain edema in animal models of stroke that receive blocking antibodies against specific cell adhesion molecules that mediate leukocyte recruitment (Yilmaz and Granger, 2008), anti-inflammatory treatment (Sharkey and Butcher, 1994), and immune deficient animals (Yilmaz et al., 2006; Hurn et al., 2007; Kleinschnitz et al., 2010; Gelderblom et al., 2012).

\section{$\alpha \beta$ T CELLS AND REGULATORY T CELLS IN STROKE}

Compared to resident microglia, infiltrating macrophages and neutrophils, lymphocytes and NK cells infiltrate the ischemic hemisphere in small numbers. Nevertheless, $\mathrm{T}$ cells have a great impact on stroke outcome. The initial observation by Yilmaz et al. that lymphocyte deficient rag1 ${ }^{-/-}$mice are protected from stroke (Yilmaz et al., 2006) could be extended to mice with severe combined immunodeficiency lacking $\mathrm{T}$ cells and B cells (Hurn et al., 2007 ) and to $\mathrm{CD}^{+}$and $\mathrm{CD} 8^{+} \mathrm{T}$ cell-deficient animals (Yilmaz et al., 2006). Direct detrimental mechanisms elicited by $\alpha \beta \mathrm{T}$ cell in stroke pathophysiology include $\mathrm{CD} 8^{+} \mathrm{T}$ cell derived perforin mediated cytotoxicity (Liesz et al., 2011) and IL-21 secreted by $\mathrm{CD}^{+} \mathrm{T}$ cells (Clarkson et al., 2014).

The classical activation of $\alpha \beta$ T cells requires several coincident signals: (1) engagement of the antigen receptor; (2) costimulatory receptors; (3) cytokine receptors such IL-2 receptor; a process requiring at least 3-5 d (Jensen et al., 2008). Multiple studies using antigen specific mucosal tolerization protocols against myelin antigens suggest the involvement of adaptive mechanism in stroke pathophysiology. Already in 1997 the group from Hallenbeck demonstrated that rodents tolerized with myelin peptides are protected from ischemic stroke (Becker et al., 1997). Mechanistically the protective effects could be attributed to IL-10 producing T cells (Frenkel et al., 2005) and transforming growth factor- $\beta 1$ (Becker et al., 2003).

These classical concepts of $\mathrm{T}$ cell activation are challenged by the observation that detrimental $\mathrm{T}$ cell dependent effects following cerebral ischemia can be observed already $24 \mathrm{~h}$ post stroke, in an antigen independent fashion (Kleinschnitz et al., 2010). Similarly controversial is the role of regulatory $T_{\text {regs }}$ and B cells in stroke. Liesz and colleagues showed that endogenous $\mathrm{T}_{\text {regs }}$ are protective in later stages following stroke when the lesions were small (Liesz et al., 2009) and that their beneficial functions depend on IL-10 (Liesz et al., 2013). However, a lot of the observed effects of $\mathrm{T}_{\text {regs }}$ cannot be attributed to concepts of adaptive immunity. For example, an early direct inhibitory effect of $\mathrm{T}_{\text {regs }}$ on the MMP9 production from neutrophils was a recently suggested mechanism (Li et al., 2013). In this model, transfer of regulatory $\mathrm{T}_{\text {regs }}$ conferred protective effects on the outcome already on day one after stroke even before $\mathrm{T}_{\text {regs }}$ infiltrated the 

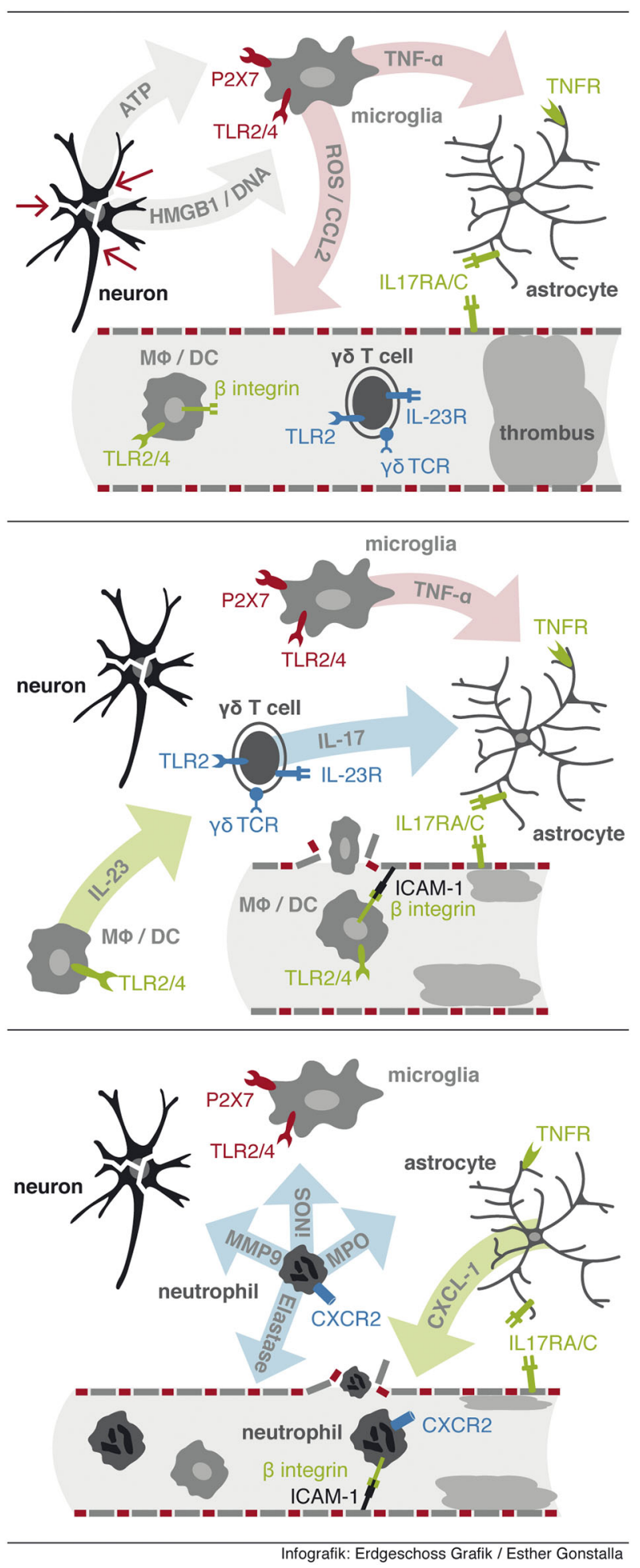

FIGURE 1 | Sequential events leading to neutrophil infiltration. First, release from DAMPs from injured cells activates resident microglia via PPRs to release proinflammatory factors including TNF- $\alpha$. Second, IL-23 activates $\gamma \delta$ T cells to rapidly secrete IL-17 in the ischemic tissue. Third, neutrophil infiltration is initiated via IL-17 and TNF- $\alpha$ synergistically induced expression of $\mathrm{CXCL}-1$ in astrocytes. ischemic brain. Protective effects could be attributed to program death-1 ligand 1 (PD-L1) dependent inhibition on MMP9 production in neutrophils in the peripheral circulation which then led to a consecutive protection of the blood brain barrier ( $\mathrm{Li}$ et al., 2014). Further studies even challenged the overall concept of $\mathrm{T}_{\text {regs }}$ as endogenous protective immune cell population in stroke (Ren et al., 2011) and a recent study suggests that $\mathrm{T}_{\text {regs }}$ have an early detrimental role, by inducing dysfunction of the cerebral microcirculation (Kleinschnitz et al., 2013). While the data on $\mathrm{T}$ cell effects in particular $\mathrm{T}_{\text {regs }}$ in stroke is still controversial, it is clear that most of the important immunological effects are not following classical concepts of adaptive immunity, suggesting an innate like behavior of lymphocytes. In this line, atypical T cells such as $\gamma \delta \mathrm{T}$ cell and NK cells are likely to participate in the early orchestration of the inflammatory reaction. For NK cells it has been shown that neuronal cell death is mediated by IFN- $\gamma$ - and Perforin-dependent pathways as early as $3 \mathrm{~h}$ post reperfusion (Gan et al., 2014). A lot more data exist on $\gamma \delta \mathrm{T}$ cell, which we will focus on in the following section.

\section{BIOLOGY OF $\gamma \delta$ T CELLS SUBPOPULATIONS}

Like $\alpha \beta$ T cells, $\gamma \delta$ T cells develop in the thymus using the recombinase activated gene product (RAG) for the somatic rearrangement of $\mathrm{V}$ (variable), D (Diversity and J (joining) gene segments of the $\gamma$ and $\delta$ chains of their T cell receptor (TcR) (reviewed in Raulet, 1989). Compared to $\alpha \beta \mathrm{TcR}$, the sets of TcR detected on $\gamma \delta \mathrm{T}$ cells are limited. Many $\gamma \delta$ subsets, primarily the ones populating certain tissues such as the epidermis, dermis, intestine, lungs and uterus are displaying an even higher limitation of their TcR diversity. These tissue-specific $\gamma \delta \mathrm{T}$ cell subsets show a biased use of certain TcR V gene segments. Since some of them express "invariant" TcRs with identical (canonical) junctional sequences, they are also named canonical $\gamma \delta \mathrm{T}$ cells. As reviewed by Vantourout and Hayday, the limited TcR diversity implies that these cells recognize either pathogen encoded antigens, that are likely to be encountered in specific tissues such as the epidermis, or self-encoded molecules that reflect a dysregulated state of that tissue (Vantourout and Hayday, 2013). Since these $\gamma \delta \mathrm{T}$ cell subsets can be rapidly activated without the requirement of prior clonal expansion they are also called "innate like" $\mathrm{T}$ cells. In contrast to canonical $\gamma \delta \mathrm{T}$ cells so-called non-canonical $\gamma \delta \mathrm{T}$ cells, which are characterized by an expression of more diverse $\gamma \delta$ TcRs, are homing into secondary lymphoid tissues. Here they make up a minor fraction of rodent and human $\mathrm{T}$ cells after birth (in mice $1-4 \%$ of all $\mathrm{T}$ cells). In the context of immune responses noncanonical $\gamma \delta \mathrm{T}$ cells are capable to participate distant from their original site of residence, by trafficking to the site of inflammation in solid organs (reviewed by Korn and Petermann, 2012). Similar to $\alpha \beta$ T cells, $\gamma \delta$ T cells can be divided by their cytokine profile. Mouse $\gamma \delta \mathrm{T}$ cells which are developing from fetal liver progenitors undergo functional pre-programming, which leads to a subpopulation of IL-17 producing Scart- $2^{+}$and CCR $6^{+} \gamma \delta$ $\mathrm{T}$ cells on one side and IFN- $\gamma$ producing NK.1.1 $1^{+}$and $\mathrm{CD} 27^{+}$ $\gamma \delta \mathrm{T}$ cells on the other side. Both subpopulations have an innate like phenotype, since they can be rapidly activated without prior clonal expansion (Vantourout and Hayday, 2013). $\gamma \delta \mathrm{T}$ cells fulfill 
important sentinel functions in the immune system. The ability of $\gamma \delta \mathrm{T}$ cells to recognize molecules that are rapidly displayed after stress without requiring extensive clonal expansion permits $\gamma \delta \mathrm{T}$ cells to participate in early stages of immune responses. In such scenarios $\gamma \delta \mathrm{T}$ cells act in parallel with cells of the innate immune system as sensors of dysregulation. $\gamma \delta \mathrm{T}$ cells may respond to classical signals of the adaptive immune system or to cytokine signals and either Toll-like receptor (TLR) or dectin stimuli in the absence of TcR ligation. Activation of the $\gamma \delta$ TcR can occur through major histocompatibility complex (MHC)-related and unrelated TcR ligands, which are including foreign- and self-antigens. This allows $\gamma \delta \mathrm{T}$ cells to respond to infection and sterile tissue dysregulation such as ischemia. Beside TcR dependent mechanisms $\gamma \delta \mathrm{T}$ cell activation can be mediated through engagement of the activating natural killer receptors (NKRs) such as NK group 2 member D (NKD2D), by patter recognition receptors including TLRs (reviewed by Bonneville et al., 2010) and through cytokines such as IL-1 $\beta$ and/or IL-23 (Sutton et al., 2009). The constitutive expression of IL-23 and IL-1 $\beta$ receptors by $\gamma \delta \mathrm{T}$ cells assures this rapid responsiveness. Within hours upon activation and without prior expansion systemic $\gamma \delta$ T cells can express high levels of effector cytokines, such as IFN- $\gamma$, IL-17, TNF- $\alpha$ and granzymes. In addition, $\gamma \delta \mathrm{T}$ cells are capable of producing numerous chemokines and regulatory factors including IL-13 and insulin-like-growth factor 1 (IGF-1), allowing them to interact with other immune cells, such as B cells and $\alpha \beta \mathrm{T}$ cells in the afferent phase of the immune response. Regarding the cellular interplay between $\gamma \delta$ $\mathrm{T}$ cells and innate immune cells neutrophils play a central role. Once activated, $\gamma \delta \mathrm{T}$ cells can stimulate the release of potent chemoattractants for neutrophils. In this respect, $\gamma \delta \mathrm{T}$ cells were recently shown to be the primary sources of the neutrophilattracting IL-17 in mouse models of infection (Shibata et al., 2007), hypersensitivity (Simonian et al., 2009) and autoimmunity (Roark et al., 2007). Often the activation of the innate immune system results in a feed back loop that increasingly stimulates $\gamma \delta$ $\mathrm{T}$ cells.

\section{$\gamma \delta$ T CELLS AS SENSORS OF TISSUE DAMAGE IN STROKE}

Stroke resembles classical features of a "sterile inflammation", which is characterized by a inflammation in response to tissue disruption without the involvement of pathogenic microorganisms (See Figure 1; Chen and Nuñez, 2010). Sterile inflammation shares similar mechanisms with inflammation during infection. Receptors essential for sensing microorganisms are collectively called pattern recognition receptors (PPRs). PRRs sense conserved structural moieties that are found in microorganisms and are often called pathogen-associated molecular patterns (PAMPs) (for review see Chen and Nuñez, 2010). Following ligand recognition these receptors activate downstream signaling pathways, such as the nuclear factor-кb (NF-кb), mitogenactivated protein kinase (MAPK) and type I interferon pathways, which result in the upregulation of pro-inflammatory cytokines and chemokines that are important in inflammatory responses. In non-infectious conditions immune cells can be activated via recognition of endogenous material by PPRs. These endogenous molecules have been named danger-associated molecular patterns (DAMPs). Under physiological conditions these DAMPs are localized intracellularly. Under conditions of apoptotic cells death, cells are cleared immunologically silent without significant release of DAMPs into the extracellular environment. In contrast, necrosis following ischemia leads to loss of cell integrity and release of the cell content into the extracellular space. DAMPs derived from necrotic cells include the chromatin-associated protein high-mobility group box 1 (HMGB1), heat shock proteins (HSPs), mitochondrial peptides and purine metabolites, such as adenosine triphosphate (ATP) and uric acid (reviewed by Chen and Nuñez, 2010 and Shen et al., 2013). Consecutively, activated receptors and signaling pathways include TLR2/4/9, CD24, CD44, NLRP3, formyl peptide receptor 1, RAGE and IL-1 receptor. In the context of stroke DAMPs are massively released into the extracellular compartment. In stroke several pathways have been described, including TLR2/4, CD38, P2X7 and RAGE, which are associated with an worsened outcome (Liu et al., 2007; Tang et al., 2008; Choe et al., 2011; Arbeloa et al., 2012). As we discussed above $\gamma \delta \mathrm{T}$ cells can be activated directly by DAMPs via TLR $1 / 2$ and dectin receptors and cytokines, such as IL-1 $\beta$ and IL-23 (Martin et al., 2009; Sutton et al., 2012). Following stroke, there is clear evidence that IL-23 activates IL-17 production in $\gamma \delta \mathrm{T}$ cells (Shichita et al., 2009). Even though it is likely that further signals via $\mathrm{TcR}$ and TLR/dectin receptors are necessary to fully activate $\gamma \delta \mathrm{T}$ cells, the actual experimental data is outstanding.

\section{EFFECTOR MECHANISMS OF $\gamma \delta$ T CELLS IN STROKE}

Several papers have shown a significant contribution of $\gamma \delta \mathrm{T}$ cells and IL-17 in stroke and other conditions of central nervous system inflammation (Kebir et al., 2007; Shichita et al., 2009; Caccamo et al., 2011; Gelderblom et al., 2012). In ischemia reperfusion injury of the brain we and others have observed a pathogenic role of $\gamma \delta \mathrm{T}$ cells, which can be detected in ischemic brain tissue as early as $6 \mathrm{~h}$ post ischemia (Shichita et al., 2009; Gelderblom et al., 2012). Effector mechanisms of $\gamma \delta \mathrm{T}$ cells in stroke primarily depend on their IL-17 production. In stroke, synergistic stimulation of astrocytes by IL-17 and TNF- $\alpha$ induces a massive induction of neutrophil attracting chemokines including CXCL-1 (Gelderblom et al., 2012), resulting in a subsequent neutrophil infiltration, which is leading to an increased tissue damage. Activated macrophages and microglia are secreting high amounts of TNF- $\alpha$ in the ischemic tissue. In the presence of the TNF- $\alpha$ rich milieu the additional IL-17 signal leads to the rapid increase of CXCL-1 via a stabilizing effect on the CXCL-1 RNA in astrocytes. Blocking either signal, IL-17 or the CXCL-1/CXCR2-axis, results in a robust reduction in infarct size and a significant improved neurological outcome. Even if an anti-IL-17 antibody is administered $6 \mathrm{~h}$ after stroke, neutrophil invasion can be blocked (Gelderblom et al., 2012). Neutrophil independent effects of IL-17 secreted by $\gamma \delta \mathrm{T}$ cells in stroke include the induction of MMP3 and MMP9 which are associated with blood brain barrier breakdown (Shichita et al., 2009; Gelderblom et al., 2012). Other potential effector functions of $\gamma \delta \mathrm{T}$ are engagement of death inducing receptors such as CD95 or TNF-related apoptosis-inducing ligand receptors (TRAILR), and the release of cytotoxic effector molecules, such 
as perforin and granzymes (See Figure 1). Molecular signals directing $\gamma \delta \mathrm{T}$ cell into the ischemic brain is another unresolved issue. $\gamma \delta \mathrm{T}$ cell subpopulations can be divided by Scart-2 and CCR6 vs. NK.1.1 and CD27 expression into IL-17 vs. IFN- $\gamma$ producing $\mathrm{T}$ cells, respectively. The functional relevance of the CCR6 expression on IL-17 producing $\gamma \delta$ T cells is supported by experimental data, showing that the migration of $\gamma \delta \mathrm{T}$ cells into the inflamed liver depends on the CCL20/CCR6 axis (Hammerich et al., 2014). Nevertheless, in stroke it is so far unclear which chemokines/chemokine receptors are essential for the entry of $\gamma \delta$ T cells into the ischemic brain and which $\gamma \delta$ T cell subpopulations are migrating into the ischemic brain.

\section{ROLE OF $\gamma \delta$ T CELLS IN HUMAN STROKE PATHOPHYSIOLOGY}

Most of the data on inflammation in stroke is derived from studies in rodent models. These models have several drawback, including differences between the immune system of rodents and humans. Further, the vast majority of stroke patients are older that 65 and are characterized by co-morbidities, which are not reflected in rodent models (Heuschmann et al., 2010). Despite these discrepancies, results from post-mortem and imaging studies in human stroke demonstrate that a rapid activation of the resident and systemic immune system are hallmarks of human stroke pathophysiology (Mena et al., 2004; Price et al., 2004; Thiel and Heiss, 2011). Similar to experimental stroke, neutrophils are recruited into the ischemic brain within $24 \mathrm{~h}$ after symptom onset (Chuaqui and Tapia, 1993; Price et al., 2004) and microglia undergo rapid activation in the infarct core but also remote areas such as fiber tracts or relay nuclei (Thiel and Heiss, 2011). These findings let to several clinical trials targeting neutrophils in human stroke. Studies employing inhibitors of the neutrophil-endothelial cell interaction including CD18 and ICAM-1 were conducted, none of them showing favorable results on the clinical outcome parameters (del Zoppo, 2010). Nevertheless, the immunological understanding of the post ischemic inflammatory response was limited when these human trials were designed. Regarding our current understanding of the stroke induced inflammation IL-17 seems to be promising target. Infiltration by $\gamma \delta \mathrm{T}$ cells and secretion of IL-17 have been demonstrated in ischemic pathological human brain tissue (Li et al., 2005; Gelderblom et al., 2012). Similarly, IL-17 induced downstream pathways can be found. The IL-17 presence in the ischemic brain is early and short-lived and has most likely only pro-inflammatory effects. Therefore a short antiIL-17 intervention could be beneficial without producing side effects, for example enhancing the systemic immune suppression. Recent data from human clinical trials with humanized neutralizing IL-17A antibodies in patients with autoimmune disease showed that treatment is well tolerized and effective (Hueber et al., 2010).

\section{SUMMARY}

Inflammation can enhance ischemic damage and lymphocytes seem to be important component of this process. Interestingly, the classical concepts of adaptive immune responses do not explain all observed effects. Several innate like features of lymphocytes dominate the early pro-inflammatory events. Particularly atypical
T cells such as $\gamma \delta$ T cells could explain some of these discrepancies and targeted treatment against their signature cytokine IL-17 might be a promising treatment option.

\section{REFERENCES}

Arbeloa, J., Pérez-Samartín, A., Gottlieb, M., and Matute, C. (2012). P2X7 receptor blockade prevents ATP excitotoxicity in neurons and reduces brain damage after ischemia. Neurobiol. Dis. 45, 954-961. doi: 10.1016/j.nbd.2011.12.014

Becker, K., Kindrick, D., McCarron, R., Hallenbeck, J., and Winn, R. (2003). Adoptive transfer of myelin basic protein-tolerized splenocytes to naive animals reduces infarct size: a role for lymphocytes in ischemic brain injury? Stroke 34, 1809-1815. doi: 10.1161/01.str.0000078308.77727.ea

Becker, K. J., McCarron, R. M., Ruetzler, C., Laban, O., Sternberg, E., Flanders, K. C., et al. (1997). Immunologic tolerance to myelin basic protein decreases stroke size after transient focal cerebral ischemia. Proc. Natl. Acad. Sci. U S A 94, 10873-10878. doi: 10.1073/pnas.94.20.10873

Bonneville, M., O’Brien, R. L., and Born, W. K. (2010). Gammadelta T cell effector functions: a blend of innate programming and acquired plasticity. Nat. Rev. Immunol. 10, 467-478. doi: 10.1038/nri2781

Caccamo, N., La Mendola, C., Orlando, V., Meraviglia, S., Todaro, M., Stassi, G., et al. (2011). Differentiation, phenotype and function of interleukin-17producing human V $\gamma 9 \mathrm{~V} \delta 2 \mathrm{~T}$ cells. Blood 118, 129-138. doi: 10.1182/blood2011-01-331298

Chen, G. Y., and Nuñez, G. (2010). Sterile inflammation: sensing and reacting to damage. Nat. Rev. Immunol. 10, 826-837. doi: 10.1038/nri2873

Choe, C.-U., Lardong, K., Gelderblom, M., Ludewig, P., Leypoldt, F., Koch-Nolte, F., et al. (2011). CD38 exacerbates focal cytokine production, postischemic inflammation and brain injury after focal cerebral ischemia. PLoS One 6:e19046. doi: 10.1371/journal.pone.0019046

Chuaqui, R., and Tapia, J. (1993). Histologic assessment of the age of recent brain infarcts in man. J. Neuropathol. Exp. Neurol. 52, 481-489. doi: 10. 1097/00005072-199309000-00006

Clarkson, B. D. S., Ling, C., Shi, Y., Harris, M. G., Rayasam, A., Sun, D., et al. (2014). $\mathrm{T}$ cell-derived interleukin (IL)-21 promotes brain injury following stroke in mice. J. Exp. Med. 211, 595-604. doi: 10.1084/jem.20131377

del Zoppo, G. J. (2010). Acute anti-inflammatory approaches to ischemic stroke. Ann. N Y Acad. Sci. 1207, 143-148. doi: 10.1111/j.1749-6632.2010. 05761.x

Frenkel, D., Huang, Z., Maron, R., Koldzic, D. N., Moskowitz, M. A., and Weiner, H. L. (2005). Neuroprotection by IL-10-producing MOG CD4+ T cells following ischemic stroke. J. Neurol. Sci. 233, 125-132. doi: 10.1016/j.jns.2005. 03.022

Gan, Y., Liu, Q., Wu, W., Yin, J. X., Bai, X. F., Shen, R., et al. (2014). Ischemic neurons recruit natural killer cells that accelerate brain infarction. Proc. Natl. Acad. Sci. U S A 111, 2704-2709. doi: 10.1073/pnas.1315943111

Gelderblom, M., Weymar, A., Bernreuther, C., Velden, J., Arunachalam, P., Steinbach, K., et al. (2012). Neutralization of the IL-17 axis diminishes neutrophil invasion and protects from ischemic stroke. Blood 120, 3793-3802. doi: 10.1182/blood-2012-02-412726

Hammerich, L., Bangen, J. M., Govaere, O., Zimmermann, H. W., Gassler, N., Huss, S., et al. (2014). Chemokine receptor CCR6-dependent accumulation of $\gamma \delta$ T cells in injured liver restricts hepatic inflammation and fibrosis. Hepatology 59, 630-642. doi: 10.1002/hep.26697

Heuschmann, P., Busse, O., Wagner, M., Endres, M., Villringer, A., Röther, J., et al. (2010). Schlaganfallhäufigkeit und Versorgung von Schlaganfallpatienten in Deutschland. Akt. Neurol. 37, 333-340. doi: 10.1055/s-0030-1248611

Hueber, W., Patel, D. D., Dryja, T., Wright, A. M., Koroleva, I., Bruin, G., et al. (2010). Effects of AIN457, a fully human antibody to Interleukin-17A, on Psoriasis, Rheumatoid Arthritis and Uveitis. Sci. Transl. Med. 2:52ra72. doi: 10. 1126/scitranslmed.3001107

Hurn, P. D., Subramanian, S., Parker, S. M., Afentoulis, M. E., Kaler, L. J., Vandenbark, A. A., et al. (2007). T- and B-cell-deficient mice with experimental stroke have reduced lesion size and inflammation. J. Cereb. Blood Flow Metab. 27, 1798-1805. doi: 10.1038/sj.jcbfm.9600482

Iadecola, C., and Anrather, J. (2011). The immunology of stroke: from mechanisms to translation. Nat. Med. 17, 796-808. doi: 10.1038/nm.2399

Jensen, K. D. C., Su, X., Shin, S., Li, L., Youssef, S., Yamasaki, S., et al. (2008). Thymic selection determines $\gamma \delta \mathrm{T}$ cell effector fate: antigen-naive cells make 
interleukin-17 and antigen-experienced cells make interferon $\gamma$. Immunity 29, 90-100. doi: 10.1016/j.immuni.2008.04.022

Kebir, H., Kreymborg, K., Ifergan, I., Dodelet-Devillers, A., Cayrol, R., Bernard, M., et al. (2007). Human TH17 lymphocytes promote blood-brain barrier disruption and central nervous system inflammation. Nat. Med. 13, 1173-1175. doi: $10.1038 / \mathrm{nm} 1651$

Kleinschnitz, C., Kraft, P., Dreykluft, A., Hagedorn, I., Göbel, K., Schuhmann, M. K., et al. (2013). Regulatory T cells are strong promoters of acute ischemic stroke in mice by inducing dysfunction of the cerebral microvasculature. Blood 121, 679-691. doi: 10.1182/blood-2012-04-426734

Kleinschnitz, C., Schwab, N., Kraft, P., Hagedorn, I., Dreykluft, A., Schwarz, T., et al. (2010). Early detrimental T-cell effects in experimental cerebral ischemia are neither related to adaptive immunity nor thrombus formation. Blood 115, 38353842. doi: 10.1182/blood-2009-10-249078

Korn, T., and Petermann, F. (2012). Development and function of interleukin 17producing $\gamma \delta$ T cells. Ann. N Y Acad. Sci. 1247, 34-45. doi: 10.1111/j.1749-6632. 2011.06355.x

Li, P., Gan, Y., Sun, B.-L., Zhang, F., Lu, B., Gao, Y., et al. (2013). Adoptive regulatory T-cell therapy protects against cerebral ischemia. Ann. Neurol. 74, 458-471. doi: 10.1002/ana.23815

Li, P., Mao, L., Liu, X., Gan, Y., Zheng, J., Thomson, A. W., et al. (2014). Essential role of program death 1-Ligand 1 in regulatory T-cell-afforded protection against blood-brain barrier damage after stroke. Stroke 45, 857-864. doi: 10. 1161/STROKEAHA.113.004100

Li, G.-Z., Zhong, D., Yang, L.-M., Sun, B., Zhongy, Z.-H., Yin, Y.-H., et al. (2005). Expression of interleukin-17 in ischemic brain tissue. Scand. J. Immunol. 62, 481-486. doi: 10.1111/j.1365-3083.2005.01683.x

Liesz, A., Suri-Payer, E., Veltkamp, C., Doerr, H., Sommer, C., Rivest, S., et al. (2009). Regulatory $\mathrm{T}$ cells are key cerebroprotective immunomodulators in acute experimental stroke. Nat. Med. 15, 192-199. doi: 10.1038/ nm. 1927

Liesz, A., Zhou, W., Mracskó, E., Karcher, S., Bauer, H., Schwarting, S., et al. (2011). Inhibition of lymphocyte trafficking shields the brain against deleterious neuroinflammation after stroke. Brain 134, 704-720. doi: 10.1093/brain/ awr008

Liesz, A., Zhou, W., Na, S. Y., Hämmerling, G. J., Garbi, N., Karcher, S., et al. (2013). Boosting regulatory $\mathrm{T}$ cells limits neuroinflammation in permanent cortical stroke. J. Neurosci. 33, 17350-17362. doi: 10.1523/JNEUROSCI.4901-12.2013

Liu, K., Mori, S., Takahashi, H. K., Tomono, Y., Wake, H., Kanke, T., et al. (2007). Anti-high mobility group box 1 monoclonal antibody ameliorates brain infarction induced by transient ischemia in rats. FASEB J. 21, 3904-3916. doi: 10. 1096/fj.07-8770com

Martin, B., Hirota, K., Cua, D. J., Stockinger, B., and Veldhoen, M. (2009). Interleukin-17-producing gammadelta $\mathrm{T}$ cells selectively expand in response to pathogen products and environmental signals. Immunity 31, 321-330. doi: 10. 1016/j.immuni.2009.06.020

Mena, H., Cadavid, D., and Rushing, E. J. (2004). Human cerebral infarct: a proposed histopathologic classification based on 137 cases. Acta Neuropathol. 108, 524-530. doi: 10.1007/s00401-004-0918-Z

Price, C. J. S., Menon, D. K., Peters, A. M., Ballinger, J. R., Barber, R. W., Balan, K. K., et al. (2004). Cerebral neutrophil recruitment, histology and outcome in acute ischemic stroke: an imaging-based study. Stroke 35, 1659-1664. doi: 10. 1161/01.str.0000130592.71028.92

Raulet, D. H. (1989). The structure, function, and molecular genetics of the gamma/delta T cell receptor. Annu. Rev. Immunol. 7, 175-207. doi: 10. 1146/annurev.immunol.7.1.175

Ren, X., Akiyoshi, K., Vandenbark, A. A., Hurn, P. D., and Offner, H. (2011). CD4+FoxP3+ regulatory T-cells in cerebral ischemic stroke. Metab. Brain Dis. 26, 87-90. doi: 10.1007/s11011-010-9226-6
Roark, C. L., French, J. D., Taylor, M. A., Bendele, A. M., Born, W. K., and O'Brien, R. L. (2007). Exacerbation of collagen-induced arthritis by oligoclonal, IL-17-producing $\gamma \delta$ T cells. J. Immunol. 179, 5576-5583. doi: 10. 4049/jimmunol.179.8.5576

Sharkey, J., and Butcher, S. P. (1994). Immunophilins mediate the neuroprotective effects of Fk506 in focal cerebral-ischemia. Nature 371, 336-339. doi: 10. $1038 / 371336 \mathrm{a} 0$

Shen, H., Kreisel, D., and Goldstein, D. R. (2013). Processes of Sterile Inflammation. J. Immunol. 191, 2857-2863. doi: 10.4049/jimmunol.1301539

Shibata, K., Yamada, H., Hara, H., Kishihara, K., and Yoshikai, Y. (2007). Resident Vdelta1+ gammadelta $\mathrm{T}$ cells control early infiltration of neutrophils after Escherichia coli infection via IL-17 production. J. Immunol. 178, 4466-4472. doi: 10.4049/jimmunol.178.7.4466

Shichita, T., Sugiyama, Y., Ooboshi, H., Sugimori, H., Nakagawa, R., Takada, I., et al. (2009). Pivotal role of cerebral interleukin-17-producing gammadeltaT cells in the delayed phase of ischemic brain injury. Nat. Med. 15, 946-950. doi: 10. 1038/nm.1999

Simonian, P. L., Roark, C. L., Wehrmann, F., Lanham, A. M., Born, W. K., O’Brien, R. L., et al. (2009). IL-17A-Expressing T Cells are essential for bacterial clearance in a Murine Model of Hypersensitivity Pneumonitis. J. Immunol. 182, 65406549. doi: 10.4049/jimmunol.0900013

Sutton, C. E., Lalor, S. J., Sweeney, C. M., Brereton, C. F., Lavelle, E. C., and Mills, K. H. G. (2009). Interleukin-1 and IL-23 induce innate IL-17 production from gammadelta $\mathrm{T}$ cells, amplifying Th17 responses and autoimmunity. Immunity 31, 331-341. doi: 10.1016/j.immuni.2009.08.001

Sutton, C. E., Mielke, L. A., and Mills, K. H. G. (2012). IL-17-producing $\gamma \delta$ T cells and innate lymphoid cells. Eur. J. Immunol. 42, 2221-2231. doi: 10.1002/eji. 201242569

Tang, S.-C., Lathia, J. D., Selvaraj, P. K., Jo, D.-G., Mughal, M. R., Cheng, A., et al. (2008). Toll-like receptor-4 mediates neuronal apoptosis induced by amyloid beta-peptide and the membrane lipid peroxidation product 4-hydroxynonenal. Exp. Neurol. 213, 114-121. doi: 10.1016/j.expneurol.2008. 05.014

Thiel, A., and Heiss, W.-D. (2011). Imaging of microglia activation in stroke. Stroke 42, 507-512. doi: 10.1161/STROKEAHA.110.598821

Vantourout, P., and Hayday, A. (2013). Six-of-the-best: unique contributions of. Nat. Rev. Immunol. 13, 88-100. doi: 10.1038/nri3384

Yilmaz, G., Arumugam, T. V., Stokes, K. Y., and Granger, D. N. (2006). Role of T lymphocytes and interferon-gamma in ischemic stroke. Circulation 113, 21052112. doi: 10.1161/circulationaha.105.593046

Yilmaz, G., and Granger, D. N. (2008). Cell adhesion molecules and ischemic stroke. Neurol. Res. 30, 783-793. doi: 10.1179/174313208X341085

Conflict of Interest Statement: The authors declare that the research was conducted in the absence of any commercial or financial relationships that could be construed as a potential conflict of interest.

Received: 20 September 2014; accepted: 17 October 2014; published online: 05 November 2014.

Citation: Gelderblom M, Arunachalam P and Magnus T (2014) $\gamma \delta$ T cells as early sensors of tissue damage and mediators of secondary neurodegeneration. Front. Cell. Neurosci. 8:368. doi: 10.3389/fncel.2014.00368

This article was submitted to the journal Frontiers in Cellular Neuroscience.

Copyright (c) 2014 Gelderblom, Arunachalam and Magnus. This is an open-access article distributed under the terms of the Creative Commons Attribution License (CC $B Y)$. The use, distribution and reproduction in other forums is permitted, provided the original author(s) or licensor are credited and that the original publication in this journal is cited, in accordance with accepted academic practice. No use, distribution or reproduction is permitted which does not comply with these terms. 